\title{
DLAs in simulated galaxies and dust obscuration
}

\section{David K. Churches, Mike G. Edmunds and Alistair H. Nelson}

School of Physics \& Astronomy, Cardiff University, Cardiff, CF2 3YB, UK

email: nelsona@cf.ac.uk

\begin{abstract}
We report here and use the results of a set of three-dimensional SPH/Treecode simulations which model the formation and early evolution of disk galaxies, including the generation of heavy elements by star formation, to investigate the effects of dust absorption in quasar absorption line systems. Using a simple prescription for the production of dust, we have compared the column density, zinc abundance, and optical depth properties of our models to the known properties of damped Lyman alpha systems. We find that a significant fraction of our model galaxy disks have a higher column density than any observed DLA system. We are also able to show that such parts of the disk tend to be optically thick, implying that any background quasar would be obscured through much of the disk. This would produce the selection effect against the denser absorption systems thought to be present in observations.
\end{abstract}

\section{Introduction}

The purposes of this work were :-

1) to investigate the plausibility of proto-galaxies as the originators of DLAs, and

2) to illuminate the effect of dust obscuration on DLA count statistics.

In order to do this we have used,

1) an existing code for resolved simulations of protogalaxy formation,

2) a model for generation of heavy elements via star formation, and

3) a model for dust in the ISM of the galaxy.

The following sections describe these three elements of the model.

\section{The model}

\subsection{The simulations}

The code which was used to perform the simulations is described in detail in Williams \& Nelson (2001), Churches et al. (2001), and Churches et al. (2004). Only a brief overview will be given here. The code is a standard SPH/Treecode (Barnes \& Hut 1986; Gingold \& Monaghan 1977; Lucy 1977) which has been augmented to include the effects of star formation. The star formation rate per unit volume is given by a Schmidt law (Schmidt, 1959),

$$
\frac{\mathrm{d} \rho_{g}}{\mathrm{~d} t}=-k \rho_{g}^{n},
$$

where $\rho_{g}$ is the gas density and $k$ and $n$ are constants. Observations by Kennicutt (1998) suggest that $n \simeq 1.5$.

We follow the approach of Katz (1991), Katz \& Gunn (1991), Katz (1992), Steinmetz \& Muller (1994), Vedel et al. (1994), and Steinmetz \& Muller (1995) which is to model the collapse of an isolated spherical perturbation. These models have a resolution which is less 

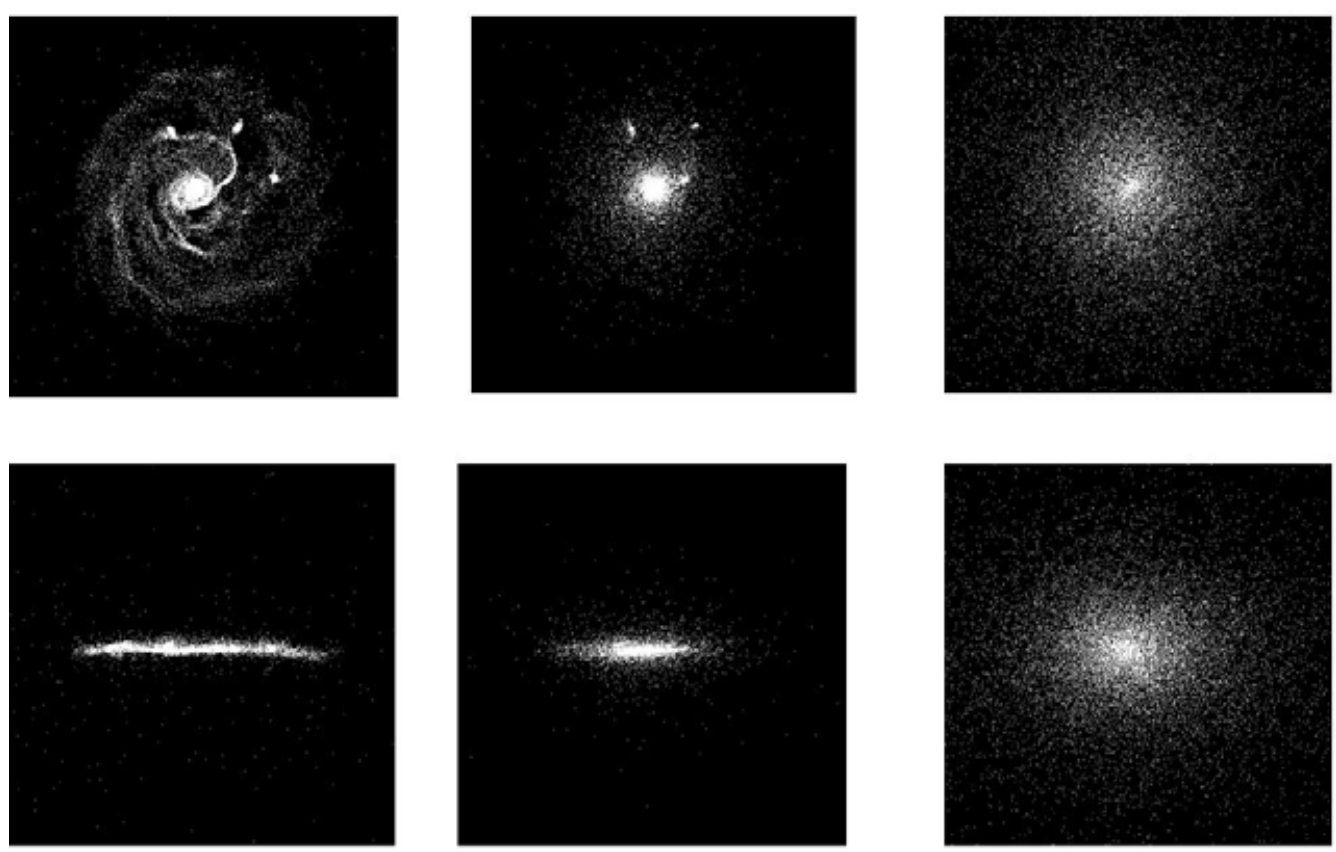

Figure 1. Final quasi-steady state of an example simulation, gas on left, stars in the middle, and dark matter on the right

than $1 \mathrm{kpc}$, and so can be used to probe internal structure, and have had great success in forming realistic disk galaxies. We start with a spherical dark matter halo which is composed of collisionless particles, and which is in solid body rotation (see below). Embedded in this are SPH gas particles which will model the gaseous component. As the simulation proceeds, gas is converted into stars, and collisionless star particles are formed.

The effect of tidal torques by neighbouring halos on the dark halo to be modelled is approximated by setting the system in solid body rotation. The amount of rotation is characterised by the dimensionless spin parameter $\lambda$, which is given by

$$
\lambda=-J|E|^{1 / 2} G^{-1} M^{5 / 2},
$$

where $J$ is the total angular momentum, $E$ the total energy, $M$ the total mass of the system, and $G$ the gravitational constant. The theoretical prediction of Peebles (1969) is that $\lambda$ is of the order of 0.08 , a value which has been confirmed by N-body experiments.

If one makes the assumption that a gas disk with a mass of $M=5 \times 10^{11} M_{\odot}$, a radius of $10 \mathrm{kpc}$, and height of $200 \mathrm{pc}$ turns $90 \%$ of its mass into stars over a timescale of $5 \mathrm{Gyr}$ with $n=1.5$, then the value for $k$ thus obtained is

$$
k=1.45 M_{\odot}^{1 / 2} \mathrm{pc}^{3 / 2} \mathrm{Gyr}^{-1}
$$

which we use here. Fig. 1 shows the final result of such a calculation (Williams \& Nelson 2001) with gas on the left, stars in the middle, dark matter on the right, and the face-on and edge-on views are on top and bottom respectively.

A much wider range of initial conditions were investigated in Churches et al. (2001) and Churches (1999). These simulations have shown that the parameters which have the most significant effect upon the final properties of the model galaxies are the total mass of the system and its angular momentum. Therefore here we present results for three 
different values of each of these two parameters. We choose three values for the total mass:

$$
M=2.5 \times 10^{11} M_{\odot}, 5 \times 10^{11} M_{\odot}, \text { and } 10^{12} M_{\odot}
$$

which were combined with $\lambda=0.06$; and for one of these masses $\left(5 \times 10^{11} M_{\odot}\right)$, we varied the initial angular momentum, producing the following values of the dimensionless spin parameter:

$$
\lambda=0.06,0.09 \text {, and } 0.12 \text {. }
$$

\subsection{Calculation of metallicities}

Chemical evolution is modelled by an extension to the well-known simple model, which takes a fixed volume of the galaxy and turns gas into stars within it. This model makes the prediction that the abundance of heavy elements within the volume varies with time via the following relation:

$$
\frac{\mathrm{d}(Z g)}{\mathrm{d} t}=(p-Z) \alpha \frac{\mathrm{d} S}{\mathrm{~d} t},
$$

where $g$ is the total mass of gas within the box, a fraction $Z$ of which is in the form of heavy elements. $d S$ is the mass of gas which has just been turned into stars in time interval $d t$, and the yield $p$ is the ratio of the mass of metals which is returned to the ISM to the mass which is lost to the stellar graveyard, per generation of star formation. The extension which we have made is to let the stars and gas de-couple from each other and evolve as dynamically separate systems, whereas in the simple model they are forced to stay together in the closed box. This is achieved by applying Eqs. 2.1 and 2.6 to each $\mathrm{SPH}$ gas particle. We then form a population of collisionless stellar particles where those gas particles with a larger star formation rate are more likely to spawn a new stellar particle, as is described fully in Churches et al. (2001).

Given that in the simulations we know both the mass $m_{i}$ and metallicity $Z_{i}$ of each SPH gas particle (and therefore the mass of metals associated with each gas particle is $m_{i} Z_{i}$ ), this is a simple calculation. We need to compare our results to observations of real damped Lyman alpha systems. However, instead of $Z$, observers usually measure the abundance of a particular heavy element $X$ with respect to hydrogen, $[X / H]$. Often $[Z n / H]$ is used as an abundance indicator because zinc is not believed to become significantly depleted onto dust grains. Therefore a measurement of $[\mathrm{Zn} / \mathrm{H}]$ for the gas is more likely to represent the true zinc abundance in the object. Pagel et al. (1992) give an estimate for the relationship between the overall heavy element abundance $Z$ and $O / H, Z=23(O / H)$. Using this, we can express the abundance of $Z n$ with respect to hydrogen, under the assumption that all the heavy elements increase in step with one another. The result is (Churches 1999)

$$
\log _{10} Z=[Z n / H]-1.77 \text {. }
$$

\subsection{A simple dust model}

We use an elementary model for the absorption properties of dust in galaxies. This model is presented in Mathlin et al. (2001) and Churches (1999), and so only a summary of the main results are given here. Our model calculates the optical depth $\tau$ which is produced by looking through a column of gas which has column density $N_{\text {gas }}$ and metallicity $Z$. We assume that a constant fraction $\eta$ of the heavy elements are in the form of dust, an assumption for which plausible physical arguments can be made (Edmunds 2001). The dust is uniformly mixed with the gas and is composed of dust particles with radius $r_{d u s t}$ and density $\rho_{\text {dust }}$. This gives

$$
\tau=\frac{3 \eta Z 1.3 m_{H} N_{\text {gas }}}{4 r_{\text {dust }} \rho_{\text {dust }}} .
$$


(a)

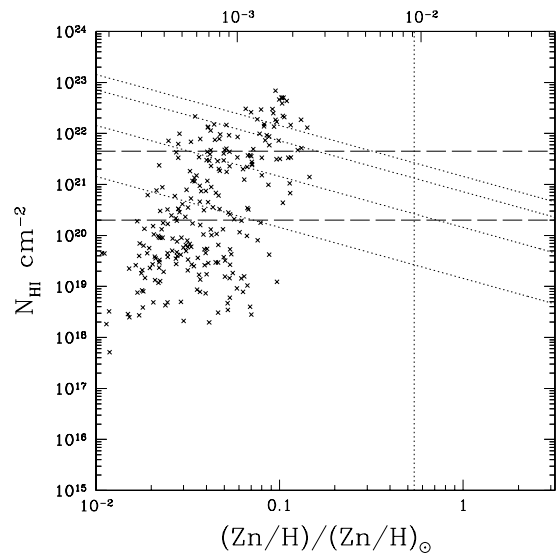

(c)

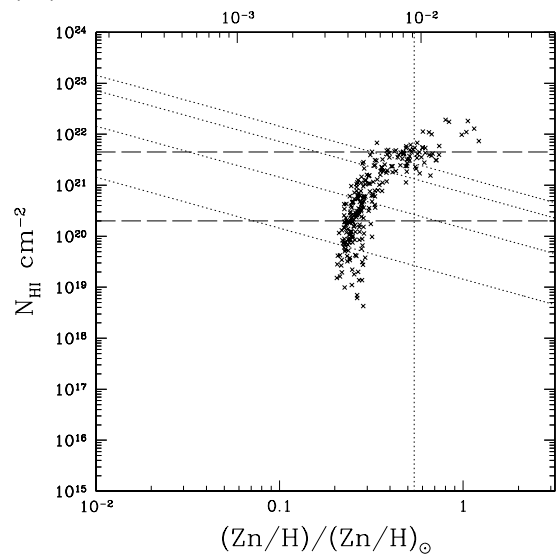

(b)

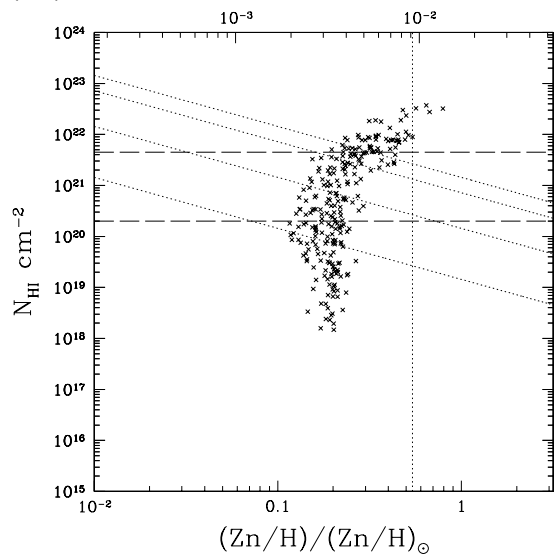

(d)

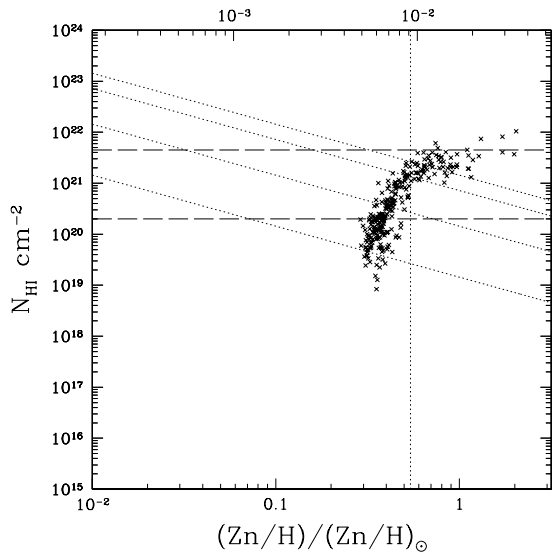

Figure 2. $N_{\mathrm{HI}}$ vs. $[\mathrm{Zn} / \mathrm{H}]$ at the 4 redshifts (a) 2.2 , (b) 1.3 , (c) 0.8 , and (d) 0.4 , for the $\left(5 \times 10^{11} M_{\odot}, \lambda=0.06\right)$ model.

For $r_{d u s t}$ and $\rho_{\text {dust }}$ we have taken $r_{d u s t}=10^{-7} \mathrm{~m}$ and $\rho_{\text {dust }}=2,000 \mathrm{~kg} \mathrm{~m}^{-3}$, and for $\eta$, Edmunds \& Eales (1998) suggest that $\eta=0.5$ is acceptable. So

$$
\tau=4.07 \times 10^{-20} \mathrm{~cm}^{2} Z N_{\text {gas }}\left(\mathrm{cm}^{-2}\right) .
$$

Using Eq. 2.9 we can put an estimate on the optical depth produced by looking through a region of a galaxy if it has metallicity $Z$ and column density $N_{\text {gas }}$. This equation may be expressed in terms of $[Z n / H]$ instead of $Z$ using Eq. 2.7.

\section{Results}

For each of the 5 pairs of model parameters the final quasi-steady galaxy disk at the end of the simulation had the column densities and Zinc abundances through vertical sight lines of the gas disks calculated, with the sight lines arranged on a $16 \times 1630 \mathrm{kpc}$ square grid. For each model these column densities and Zinc abundances were calculated at 4 times from the start of the calculation, and Fig. 2 shows the results, with $N_{\text {HI }}$ plotted 

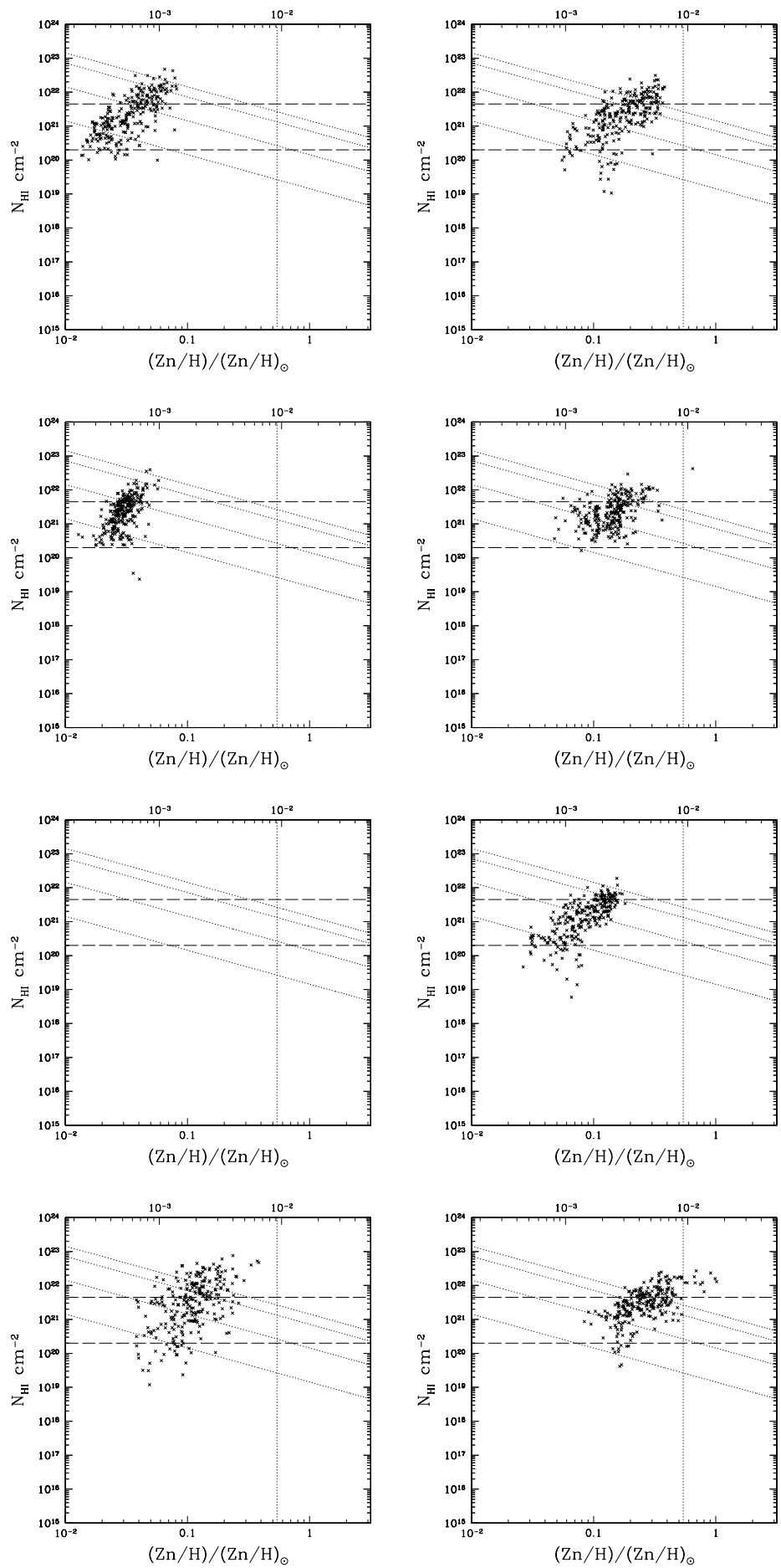

Figure 3. $N_{\mathrm{HI}}$ vs. $[\mathrm{Zn} / H]$ for the 4 other models for redshifts 2.2 (left) and 1.3 (right). The axes and scales are the same as in Fig. 2. From top to bottom, the simulation parameters are $\left(5 \times 10^{11} M_{\odot}, \lambda=0.09\right),\left(5 \times 10^{11} M_{\odot}, \lambda=0.12\right),\left(2.5 \times 10^{11} M_{\odot}, \lambda=0.09\right)$, and $\left(10^{12} M_{\odot}\right.$, $\lambda=0.09)$. 
against $Z n$ in a standard form (Mathlin et al. 2001), for the $\left(5 \times 10^{11} M_{\odot}, \lambda=0.06\right)$ model at the 4 times.

The general trend is for $N_{\mathrm{HI}}$ to decrease as time progresses, due to gas being converted to stars, while $[Z n / H]$ increases as star formation creates heavy elements. The 2 horizontal lines in Fig. 2 show the lower limit of $N_{\mathrm{HI}}$ defining a DLA $\left(2 \times 10^{20} \mathrm{HI} \mathrm{cm}^{-2}\right)$, and the largest $N_{\mathrm{HI}}$ value measured for a DLA to date. The 4 sloping lines, represent lines of increasing optical depth in dust, with values $0.01,0.1,0.5$, and 1.0 increasing upwards. Note that the simulations represent only total gas, so that the column densities here represent an upper limit for HI. No account is taken of the possibility of significant quantities of hydrogen being in the molecular form.

Fig. 3 shows the $N_{\mathrm{HI}}$ vs. $[\mathrm{Zn} / H]$ results for the other 4 cases at redshifts 2.2 and 1.3 (left and right respectively). In the lower mass case no star formation has occurred at $\mathrm{z}=$ 2.2 , so the heavy element abundance is zero, and at $\mathrm{z}=1.3$, the abundance levels are lower than for the $5 \times 10^{11} M_{\odot}$ case. While for the higher mass case the opposite trend is seen, with more heavy elements at both times. The trend of higher spread in $N_{\mathrm{HI}}$ for lower spin parameter is due to the fact that the lower angular momentum case fragments more than for the higher angular momentum, leading to a larger range of density values in the proto-galactic disk.

In general the range of values in these models overlap the observational data (Mathlin et al. 2001), but occupy a larger region, with many high density/high metallicity sight lines. But these have $\tau>0.5$, which means that they will not be observed (Pei \& Fall 1995). This trend is magnified when sight-lines inclined to the normal to the plane are considered, with higher column densities corresponding to higher optical depths.

\section{Conclusions}

The results support the idea that DLAs originate in galaxy disks at different stages of evolution. However any observational survey which counts the number of DLAs needs to recognise that a significant fraction of them may not have been detected due to dust obscuration.

\section{References}

Barnes, J., Hut, P., 1986, Nature, 324, 446

Churches, D. K., 1999, PhD thesis, Cardiff University

Churches, D. K., Nelson, A. H., Edmunds, M. G., 2001, MNRAS, 327, 610

Churches, D. K., Nelson, A. H., Edmunds, M. G., 2004, MNRAS, 347, 1234

Edmunds, M. G., 2001, MNRAS, 328, 223

Edmunds, M. G., Eales, S. A., 1998, MNRAS, 299, L29

Gingold, R. A., Monaghan, J. J., 1977, MNRAS, 181, 375

Katz, N., 1991, ApJ, 368, 325

Katz, N., 1992, ApJ, 391, 502

Katz, N., Gunn, J. E., 1991, ApJ, 377, 365

Kennicutt, R. C., 1998, ApJ, 498, 541

Lucy, L. B., 1977, AJ, 82, 1013

Mathlin, G. P., Baker, A. C., Churches, D. K., Edmunds, M. G., 2001, MNRAS, 321, 743

Pei, Y. C., Fall, S. M., 1995, ApJ, 454, 69

Schmidt, M., 1959, ApJ, 129, 243

Steinmetz, M., Muller, E., 1994, A\&A, 281, L97

Steinmetz, M., Muller, E., 1995, MNRAS, 276, 549

Vedel, H., Hellsten, U., Sommer-Larsen, J., 1994, MNRAS, 271, 743

Williams, P. R., Nelson, A. H., 2001, A\&A, 374, 839 